\title{
Rapid quantification of underivatized alloisoleucine and argininosuccinate using mixed-mode chromatography with tandem mass spectrometry
}

Chandler Griffin ${ }^{\mathrm{a}}$, Zineb Ammous ${ }^{\mathrm{b}}$, Gail H. Vance ${ }^{\mathrm{a}}$, Brett H. Graham ${ }^{\mathrm{a}}$, Marcus J. Miller ${ }^{\mathrm{a}^{*}}$

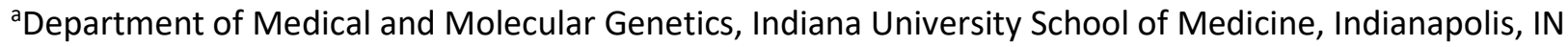
46202

${ }^{\mathrm{b}}$ The Community Health Clinic, Topeka, Indiana 46571

*Correspondence should be addressed to:

Marcus J. Miller PhD, FACMG

Department of Medical and Molecular Genetics

Indiana University School of Medicine

975 W. Walnut St., IB-344C

Indianapolis, IN 46202

(317) $278-1140$

majamill@IU.edu

This is the author's manuscript of the article published in final edited form as:

Griffin, C., Ammous, Z., Vance, G. H., Graham, B. H., \& Miller, M. J. (2019). Rapid quantification of underivatized alloisoleucine and argininosuccinate using mixed-mode chromatography with tandem mass spectrometry. Journal of Chromatography B, 1128, 121786. https://doi.org/10.1016/j.jchromb.2019.121786 


\section{ABSTRACT:}

Plasma elevations of the amino acids alloisoleucine and argininosuccinic acid (ASA) are pathognomonic for maple syrup urine disease and argininosuccinate lyase deficiency, respectively. Reliable detection of these biomarkers is typically achieved using methods with tedious sample preparations or long chromatographic separations, and many published amino acid assays report poor specificity and/or sensitivity for one or both of these compounds. This report describes a novel liquid chromatography tandem mass spectrometry (LC-MS/MS) method that provides rapid quantification of alloisoleucine and ASA in human plasma. The basis of this method is a mixed-mode solid phase separation that achieves baseline resolution of alloisoleucine from isobaric interferents without the use of derivatization or ion pairing agents. The inject-to-inject time is 6 minutes including elution, column washing and reequilibration. Validation studies demonstrate excellent limits of quantification $(1 \mu \mathrm{mol} / \mathrm{L})$, linearity $(r=$ 0.999 from $1-250 \mu \mathrm{mol} / \mathrm{L}$ ), accuracy (bias $=-3.8 \%$ and $-10.1 \%$ ), and inter-assay imprecision $(\mathrm{CV}<8.06 \%$ ) for plasma analyses. Data from long-term clinical application confirms chromatographic consistency equivalent to more traditional reversed-phase or HILIC based columns. Additional matrix studies indicate low suppression $(<10 \%)$ for a wide range of amino acids and compatibility with other matrixes such as blood spot analyses. Finally, analysis of our first 257 clinical specimens demonstrates high analytic specificity and sensitivity, allowing the detection of subtle but clinically relevant elevations of alloisoleucine and ASA that may be missed by other less sensitive methods. In conclusion, the novel LCMS/MS method reported here overcomes a number of the challenges associated with alloisoleucine and ASA quantification. Combining this approach with published incomplete amino acid quantification methods allows, for the first time, a rapid and comprehensive LC-MS/MS analysis of underivatized amino acids without the use of ion pairing agents.

\section{Key Words:}

L-alloisoleucine, ASA, intrada, amino acid, MSUD, argininosuccinic aciduria

\section{Abbreviations:}

ASA argininosuccinic acid

LC-MS/MS liquid chromatography tandem mass spectrometry

HILIC hydrophilic interaction liquid chromatography

$\mathrm{PCl}$ post column infusion 


\section{Introduction}

Quantification of plasma amino acids is a key step in the detection and management of numerous inborn errors of metabolism. Traditionally, testing for the $\sim 40$ clinically relevant amino acids in human plasma has been completed using cation exchange chromatography with post column ninhydrin derivatization and spectrophotometric detection [1].

In the last decade, liquid chromatography tandem mass spectrometry (LC-MS/MS) approaches to amino acid quantification have been adopted by some biochemical genetics laboratories [2], with the added benefits of increased analyte specificity and much quicker analysis times compared to traditional testing. Currently, most clinically validated LC-MS/MS methods rely on reversed-phase chromatography in combination with ion pairing agents (e.g., heptafluorobutryic acid) to separate and quantify derivatized $[3,4]$ or underivatized amino acids $[5,6]$. Although these approaches offer substantial improvements over traditional testing, these LC-MS/MS methods have not been widely adopted in part because (i) ion pairing agents can act as highly retentive contaminates that interfere with ionization and chromatographic separations and (ii) derivatization is costly and time consuming.

To overcome these limitations, a hydrophilic interaction liquid chromatography (HILIC) LC-MS/MS method was developed which provides excellent retention and detection of many amino acids in human plasma without the use of ion pairing agents or derivatization [7]. However, this method cannot chromatographically resolve alloisoleucine from the isomeric interferent isoleucine. In addition, in our lab's experience, argininosuccinic acid (ASA) cannot be reliably quantified due to poor peak shape and substantial carryover when using a HILIC amide column.

Alloisoleucine and ASA are clinically important amino acids that pose technical challenges when analyzed by conventional methods. Both compounds are normally found at low or undetectable levels in the plasma of healthy individuals, but when elevated, are considered pathognomonic for inherited metabolic diseases: maple syrup urine disease (MSUD; OMIM\# 248600) in the case of alloisoleucine and argininosuccinic acid lyase deficiency (ASL; OMIM\# 207900) in the case of argininosuccinic acid. Limitations in the quantification of ASA and/or alloisoleucine have been reported for other methods and are not unique to HILIC-based LC-MS/MS approaches. These include (i) poor sensitivity and specificity for ASA using traditional cation exchange chromatography analyses [8], (ii) ASA coelution with ethanolamine by ultra performance liquid chromatography [9], (iii) poor alloisoleucine resolution from isobaric compounds following derivatization and reversed phase LC-MS/MS [4]. In addition, flow 
injection methods employed by newborn screening labs can not reliably quantify either ASA or alloisoleucine. For many laboratories, it is therefore necessary to use a second complementary method to quantify ASA and/or alloisoleucine when applying existing methods to study amino acids.

Chromatographic columns that combine two or more retention mechanisms (termed "mixed-mode" columns) have emerged as an alternative for LC-MS/MS analysis of amino acids $[10,11]$. Some mixedmode columns include MS compatible ion exchange binding properties, which may be useful in the chromatographic resolution of isomeric compounds such as alloisoleucine and isoleucine. However, there remain concerns about the application of mixed-mode chromatography in routine clinical diagnostic use, particular as it relates to lengthy column washing/equilibration steps and long term chromatographic stability after exposure to a high volume of heterogeneous clinical specimens. In the following, we report the development of a rapid mixed-mode chromatography-based LC-MS/MS method to quantify alloisoleucine and ASA and we chronical this method's performance characteristics and long-term durability when integrated into routine clinical diagnostic testing.

\section{Materials and methods}

\subsection{Reagents}

High purity calibration materials were purchased from two sources and analyzed to confirm equivalency: argininosuccinic acid disodium salt hydrate (Sigma-Aldrich and Santa Cruz Biotechnology) and Lalloisoleucine (Sigma-Aldrich and Acros Organics). Additional stocks of lyophilized 4-hydroxyproline, leucine, isoleucine, delta-aminolevulinate, and arginine were purchased from Sigma Aldrich.

Isotopes tyrosine-13C6 and argininosuccinate-15N4, 13C6 were purchased from Cambridge Isotope. Tyrosine-13C6 powder was resuspended in $2 \%$ sulfosalicylic acid to a final concentration of $100 \mu \mathrm{M}$ (IS_1). Argininosuccinate-15N4, $13 \mathrm{C} 6$ was resuspended in water to a final concentration of $10 \mu \mathrm{M}$ (IS_2). Isotopes used in matrix studies include phenylalanine-13C6 (Phe), tyrosine-13C6 (Tyr), glycine15N (Gly), isoleucine-13C6 (Iso), tryptophan-indole-D5 (Trp), histdine-D3 (His) and argininosuccinate15N4, 13 C6 (Asa) purchased from Cambridge isotope and DL-2-Piperidine-carboxylic acid-D9 (Pip) purchase from CDN Isotopes. Isotope solutions were stored at $-20^{\circ} \mathrm{C}$ prior to use.

Optima LC-MS grade water, formic acid and acetonitrile (Fisher Scientific) and bio ultra-grade $10 \mathrm{M}$ ammonium formate (Sigma) were used in all solutions.

\subsection{Sample preparation}


Sample collection procedures were approved by the Indiana University Institutional Review Board (Protocol \#1804038720). Our sample set included specimens from individuals with the following clinical indications: hyperammonemia, hyperlactatemia, severe ketosis, failure to thrive, developmental delay, seizures, renal failure, and liver failure.

For the analysis of heparinized plasma, $40 \mu \mathrm{L}$ of specimen was mixed with $30 \mu \mathrm{L}$ each of IS_1 and IS_2 and then deproteinated by adding $700 \mu \mathrm{L}$ of solvent $\mathrm{A}$ ( $90 \%$ acetonitrile, $10 \mathrm{mM}$ ammonium formate, $0.15 \%$ formic acid). Samples were vortexed for $\sim 5$ seconds and then centrifuged at 15,000 rpm for 5 minutes in a microcentrifuge. Clarified supernatant was transferred to a glass auto-sampler vial for LCMS/MS analysis.

Control specimens were generated by pooling residual heparinized plasma specimens and spiking with pure ASA and alloisoleucine solutions. Controls were stored at $-20^{\circ} \mathrm{C}$ in single use aliquots.

For calibration material, pure ASA and alloisoleucine stocks were prepared individually in water and 0.1 N HCL, respectively. Immediately prior to use, ASA and alloisoleucine calibration materials were combined and diluted with water to generate solutions of the following concentration $(0.5,1,2.5,10$, $25,50,100,250 \mu \mathrm{M})$. Forty microliters of each solution were then mixed with $30 \mu \mathrm{L}$ each of IS_1 and IS_2 and $700 \mu \mathrm{L}$ of solvent A.

RECIPE ClinCheck plasma amino acid controls (IRIS Technologies International) and European Research Network for evaluation and improvement of screening, Diagnosis and treatment of Inherited disorders of Metabolism (ERNDIM; http://www.erndim.org/) 2018 amino acids in serum scheme proficiency testing materials were resuspended according to the manufacturer's guidelines.

\subsection{LC-MS/MS analysis}

Liquid chromatography was performed using an Aquity i-class UPLC system (Waters) equipped with an intrada amino acid column ( $50 \mathrm{~mm} \times 2 \mathrm{~mm}$, particle size $=3 \mu \mathrm{m}$; Imtakt) maintained at $35^{\circ} \mathrm{C}$. Mobile phase $\mathrm{A}$ ( $20 \%$ acetonitrile, $0.3 \%$ formic acid, $\mathrm{pH}^{\sim 2.4}$ ) was prepared by mixing $1.5 \mathrm{mLs}$ of formic acid with $398.5 \mathrm{mLs}$ of water and then adding $100 \mathrm{mLs}$ of acetonitrile. Mobile phase B (20\% acetonitrile, $80 \mathrm{mM}$ ammonium formate, $\mathrm{pH} \sim 6.3$ ) was prepared by mixing $396 \mathrm{mLs}$ of water and $4 \mathrm{mLs}$ of ammonium formate and then slowing adding $100 \mathrm{mLs}$ of acetonitrile with constant stirring. All mobile phase solutions were filtered through a $0.2 \mu \mathrm{m}$ nylon filter prior to use and expired after 1 week. Filtering and frequent replacement proved to be critical to avoid binary solvent manager over-pressurization, possibly due to buffer precipitation or bacterial contamination. 
Two microliters of specimen were injected into the LC system and chromatographic separation was achieved at a flow rate $0.4 \mathrm{~mL} / \mathrm{min}$ using a gradient of mobile phase $A$ and $B$ as follows: (i) $25 \%$ mobile phase B from 0-2 minutes, (ii) linear gradient increase to 99\% B from 2-3 minutes, (iii) hold at 99\% B from 3-4.5 minutes, (iv) equilibration at starting conditions ( $25 \%$ B) from 4.5-6 minutes. At the end of each batch the system was flushed with 20-80\% acetonitrile mobile phase containing low buffer content ( $<10 \mathrm{mM}$ ammonium formate) to purge the high ammonium formate containing mobile phase $b$ from the binary solvent manager.

Mass spectrometry analysis was completed by multiple reaction monitoring (MRM) on a Xevo TQS micro (Waters) in ESI positive mode using the following tune parameters: capillary voltage $=0.25 \mathrm{kV}$, desolvation temperature $550^{\circ} \mathrm{C}$, cone gas $=50 \mathrm{~L} / \mathrm{hr}$, desolvation gas $=1000 \mathrm{~L} / \mathrm{hr}$. Analyte specific tune parameters (Table 1 ) were optimized by infusing solutions of pure compounds.

All data analyses were completed using TargetLynx software (Waters). Peaks were integrated using the apex track function and quantified by comparing the response to that of a linear calibration curve that excluded the origin.

\subsection{Matrix Effects Studies}

Post column infusion was completed with a solution containing $100 \mu \mathrm{M}$ of alloisoleucine and ASA in solvent $A$ infused at $5 \mu \mathrm{l} / \mathrm{min}$. Infusion studies were completed using heparinized plasma specimens prepared following the above listed guidelines and on blank specimens (solvent A).

The combination of both suppression and analyte recovery was also explored by comparing isotope integrated intensity values in three plasma specimens vs three blanks. The percent difference was calculated as $100 \times$ (signal in matrix/ signal in blank)-1) and the average percent difference was plotted.

Spiked recovery studies were completed using three residual plasma specimens. For each specimen two different ASA spikes were completed, (i) prior to sample preparation and (ii) after protein precipitation immediately prior to MS analysis. The same spike assays were completed using water in place of matrix. All analyses were completed in duplicate. Recoveries were determined by comparing the average ASA signal in matrix compared to the water blank.

\subsection{Method Validation}

The limit of quantification was determined by studying a dilution series of calibrator material and identifying the lowest point that met the following criteria, (i) fit to the linear model (residual $<20 \%$ ), (ii) 
signal to noise $>10$ and (iii) intra-assay imprecision $<15 \%$ (calculated by studying 6 replicates). ASA LOQ was multiplied by $2 X$ to partially account for predicted matrix effects.

Accuracy was studied by spiking 4 different plasma samples each with 4 different concentrations of pure ASA or alloisoleucine to achieve a concentration increase of $28,56,112$, and $225 \mu \mathrm{M}$. Unspiked specimens were also studied and all plasma specimens were shown to have low $(<2 \mu \mathrm{M})$ or undetectable levels of endogenous ASA or alloisoleucine. Accuracy was determined by plotting all observed concentrations against the expected concentration and calculating the slope of the linear model and Pearson's correlation ( $r$ ). In addition, bias was calculated by determining the overall average percent difference between observed and expected values.

Carry-over was measured by monitoring the signal in a blank specimen analyzed immediately following the analysis of the highest standard curve point. Intra-assay imprecision was determined by analyzing control materials six consecutive times each within the same batch. Inter-assay imprecision was determined by analyzing aliquots of control samples in eight independent batches run on eight different days. Imprecision is reported in terms of the coefficient of variation (CV). The linear range of detection was determined by analysis of calibration materials and determining the range in which all points had a residual $<20 \%$, and the correlation coefficient $\left(r^{2}\right)$ was $\geq 0.98$. Samples with values outside of this range were diluted and reanalyzed.

\section{Results}

\subsection{Chromatographic separation}

A chromatographic method was developed to allow the rapid quantification of alloisoleucine and ASA by LC-MS/MS without specimen derivatization or the use of ion pairing agents. The method was based on an ammonium formate gradient elution paired with a mixed-mode solid phase $(50 \times 2 \mathrm{~mm}$ intrada column) that is reported by the manufacturer to have properties of both a normal phase and an ion exchange column. Complete baseline separation of alloisoleucine was achieved from isobaric interferents isoleucine, leucine, 4- hydroxyproline, and delta-aminolevulinic acid (Fig. 1). This method also generated good peak shape and retention for ASA and provided complete elution of strongly retained basic amino acids (e.g., arginine; Fig. 1). The total inject-to-inject time was 6 minutes including the gradient elution, washing, and re-equilibration steps.

Chromatographic separations were consistent across multiple matrixes. Figure $2 A$ and $B$ shows typical plasma alloisoleucine and ASA quantitative results from a patient with MSUD and ASL deficiency, 
respectively. During method development neat solutions, plasma, urine, and blood spot card eluates were all extensively tested and found to be essentially identical in terms of analyte retention time and peak shape.

\subsection{Suppression}

Plasma matrix suppression was explored using two complementary approaches. A post column infusion $(\mathrm{PCl})$ study found that a major region of suppression occurred early in the elution ( $R T=0.6 \mathrm{mins}$ ) and did not overlap with our analytes of interest (Fig. 3A and B). Suppression was further studied by comparing isotopic signal intensities in blank solvent vs plasma samples. Consistent with $\mathrm{PCl}$ studies, matrix induced signal loss was found to be low $(<10 \%)$ for a wide range of analytes, including those flanking

alloisoleucine (isoleucine and tyrosine) thus suggesting that surrogate internal standards may be feasible for alloisoleucine quantification (Fig. 3C).

Surprisingly, unlike the other amino acids in our study, ASA signal was significantly diminished when measured in plasma (Fig. $3 \mathrm{C}$ ). Our PCl results suggested that this observation could not be explained by suppression alone and instead raised the possibility of substantial ASA loss during preparation of plasma. To test this, we performed ASA spike recovery assays on three plasma specimens. Analyte recoveries were $105 \%$ (range $=104 \%-106 \%$ ) when ASA was added to clarified lysate following protein precipitation, but only $67 \%$ (range $=64 \%-72 \%$ ) when ASA was added to the initial plasma specimen, thus indicating a portion of endogenous ASA is lost during deproteinization. The addition of an ASA isotopic internal standard, prior to protein precipitation, was able to faithfully account for analyte loss and was therefore used in all subsequent validation studies.

\subsection{Method validation}

Results for validation studies are summarized in Table 2. Imprecision, accuracy, linearity, and carryover were all within acceptable limits. Importantly, limits of quantification (LOQ) were well below the range of clinically affected individuals (see materials and methods section 2.5 for LOQ criteria). ASA carryover was undetectable even when studying matrix samples containing outlier elevations of ASA.

Method accuracy was further supported by external calibration studies. For alloisoleucine, we analyzed RECIPE clincheck plasma reference materials level I and II and found our results were concordant with the reported value (observed vs reported, level I = 17 vs $19 \mu \mathrm{M}$ and level II = 171 vs $186 \mu \mathrm{M}$ ). For ASA, we analyzed ERNDIM quantitative schemes amino acids (serum) 2018 residual proficiency testing (PT) materials. Our results were concordant (within $+/-1$ standard deviation) with the reported median 
participant value (observed vs reported, 25 vs 32,10 vs 13,2 vs 3,60 vs 73,9 vs 13,26 vs 33,4 vs 3 , and 69 vs78 $\mu \mathrm{M}$.) The overall negative bias (-16.77\%) found in this study may reflect ASA degradation related to the age of PT specimens at the time of our analysis ( $\sim 1.5$ years past their intended use date). Notably, our in-house accuracy studies used freshly prepared ASA-spiked plasma specimens and found much lower bias $(-3.8 \%)$.

Argininosuccinic acid is known to be labile, forming ASA anhydrides under acidic conditions [12]. The stability of processed samples was studied by reanalysis of calibrators and eight different matrix samples when stored on the auto-sampler at $10^{\circ} \mathrm{C}$ for 3 days. Over this time span, plasma ASA values changed on average by $8.46 \%$.

\subsection{Solid phase stability over prolonged use}

Long-term chromatographic performance was monitored on a single column used for 62 independent batches of clinical amino acid analysis over 5 months on $~ 700$ matrix injections. During this time, the following parameters remained essentially unchanged: column backpressure, peak shape, and resolution of alloisoleucine from isoleucine/leucine. The retention time drifted lower by 0.1 mins for alloisoleucine/leucine/isoleucine, with the retention time for alloisoleucine having a CV $=3.18 \%$ (Fig. 4A). The retention time drift for ASA was much less, $C V=0.44 \%$ (Fig. 4B).

\subsection{Analysis of patient samples}

Figures $5 \mathrm{~A}$ and $\mathrm{B}$ show the results from the clinical application of this method to the first 257 plasma specimens sent sequentially to our laboratory for clinical amino acid analysis. Striking alloisoleucine elevations (> $100 \mu \mathrm{M}$ ) were noted in all patients studied with MSUD but were not detected in any of the remaining 252 samples studied that were derived from patients that either had no diagnosis or had a diagnosis of a different inborn error of metabolism (termed "other", Fig. 5A). Low levels of alloisoleucine were detectable in the majority of the "other" population including the detection of mild elevations ( $\geq 4 \mu \mathrm{M}$ ) in two patients with isovaleric acidemia (OMIM\# 243500) and one patient with 3hydroxy-3-methylglutaryl (HMG) coA lyase deficiency (OMIM\# 246450). Subtle alloisoleucine elevations have been previously reported in patients with isovaleric acidemia [13] but they may be missed by conventional methods used to quantify amino acids.

As expected, ASA was clearly elevated in all patients with argininosuccinic acid lyase (ASL) deficiency and ASA was not detectable in any individual unaffected by ASL deficiency $(n=249)$, with one exception (Fig. 5B). Trace levels of ASA (1.4 $\mu \mathrm{M})$ were identified in a patient with ornithine transcarbamylase (OTC) 
deficiency (OMIM\# 311250). Concurrent plasma elevations of arginine (236 $\mu \mathrm{M}$, normal range $=32-127$ $\mu \mathrm{M})$ and citrulline $(398 \mu \mathrm{M}$, normal range $=12-48 \mu \mathrm{M})$ were also detected in this individual, consistent with the common amino acid supplementation therapy used to treat OTC deficiency. Citrulline is the metabolic precursor to ASA whereas arginine is an ASA breakdown product. It is possible high levels of supplementation with these amino acids is causing abnormal accumulation of ASA in this individual. ASA accumulation driven by arginine supplementation has been previously reported in patients with ASL deficiency but never in an individual with functional argininosuccinic acid lyase activity, to our knowledge [14].

Idiopathic elevations of alloisoleucine and ASA were not detected in our sample set, thus indicating high analytic specificity. Included in this analysis were specimens from individuals on the following medications/supplements at the time of sampling: total parenteral nutrition, oral/intravenous Lcarnitine, Depakote/valproic acid, Keppra/levetiracetam, phenylbutyrate, gabapentin, sodium benzoate, and phenobarbital.

\section{Discussion}

This study describes a novel LC-MS/MS method for quantifying alloisoleucine and ASA that has a number of advantages over existing approaches. Foremost, to our knowledge, this is the quickest reported method for full baseline resolution of alloisoleucine and the only LC-MS/MS method for alloisoleucine measurement that does not require ion pairing agents. The latter is likely to be an attractive feature for smaller laboratories that are unable to dedicate entire LC-MS/MS systems to ion pairing based applications. The unequivocal and sensitive detection of ASA and alloisoleucine provided by this method is also likely to be of use to laboratories employing other methods where detection of these analytes is challenged by coeluting interfering compounds or insufficient limits of detection.

Our laboratory developed this method to be used as a complement to a HILIC-based LC-MS/MS approach (modified from [7]). Because sample preparation is identical between these two approaches, these methods can be easily combined to quantify the full range of clinically important amino acids using a single sample. We use a basic multi-column manager system to automate column switching and repeat sample analyses.

Our primary concern at the outset of this study was the durability of this method when used over an extended period of time and on large numbers of heterogeneous clinical specimens. During the early stages of development, we experienced numerous LC system over-pressurization events with the 
blockage occurring where the mobile phase enters the injector valve. Over-pressurization often occurred during the initial inlet startup (prior to sample analysis) thus making sample contamination an unlikely cause. Instead, we presume the blockages were related to the high ammonium formate concentration in mobile phase B. Consistent with this, over-pressurization issues completely resolved after (i) switching to an inlet method based on a constant level of acetonitrile, (ii) purging the LC system at the end of each batch, and (iii) filtering mobile phase solutions (see Methods section for more details). With these changes, we have seen consistent chromatographic results over long-term use.

In summary, we report a simple, rapid, and robust method to quantify alloisoleucine and argininosuccinic acid. Future studies are needed to expand this method to the full list of amino acids included in a standard clinical panel. The success of this method is based on the application of a novel mixed-mode column. Such solid phase chemistries are ideally suited to the analysis of the diverse groups of small polar molecules that comprise the majority of human metabolites. As our experience with mixed-mode separations grows, it seems likely that they will become more widely adopted for the study of inborn errors of metabolism.

\section{Acknowledgments}

We would like to acknowledge Kyle Adkins for his efforts to compile our clinical database and Dustin Austin for providing useful technical suggestions related to the intrada column.

The authors acknowledge the use of data derived from ERNDIM External Quality Assurance (EQA) materials in this manuscript. The use of ERNDIM EQA materials does not imply that ERNDIM endorses the methods used or the scientific validity of the findings in this manuscript. ERNDIM (www.erndim.org) is an independent, not-for-profit foundation that provides EQA schemes in the field of inborn errors of metabolism with the aim of improving diagnosis, treatment and monitoring of inherited metabolic diseases. 
Table 1 Mass Spectrometry Parameters

\begin{tabular}{lcccc}
\hline Analyte & Class* & Transition & Cone (V) & $\begin{array}{c}\text { Collision } \\
\text { (V) }\end{array}$ \\
\hline Alloisoleucine & Q & $131.968>69.055$ & 18 & 16 \\
Alloisoleucine & S & $131.968>86.033$ & 24 & 8 \\
Tyrosine_6C13 & IS & $188.100>142.110$ & 26 & 12 \\
ASA & Q & $291.160>70.071$ & 46 & 34 \\
ASA & S & $291.104>115.939$ & 44 & 22 \\
ASA_6C13_4N15 & IS & $301.132>75.027$ & 4 & 34 \\
\hline
\end{tabular}

$* \mathrm{Q}=$ transition used for quantification, $\mathrm{S}=$ supplemental transition used for confirmation, $\mathrm{IS}=$ internal standard 
Table 2 Validation Summary

\begin{tabular}{|c|c|c|c|c|c|c|c|c|c|c|}
\hline \multirow[b]{2}{*}{ Analyte } & \multirow[b]{2}{*}{$\begin{array}{l}\text { Carry- } \\
\text { over }\end{array}$} & \multirow[b]{2}{*}{$\begin{array}{l}\text { LOQ } \\
(\mu \mathrm{M})\end{array}$} & \multirow[b]{2}{*}{ Linear range ( $r$ ) } & \multicolumn{2}{|c|}{$\begin{array}{l}\text { Intra-assay } \\
\text { imprecision* }\end{array}$} & \multicolumn{2}{|c|}{$\begin{array}{c}\text { Inter-assay } \\
\text { imprecision* }\end{array}$} & \multicolumn{3}{|c|}{ Accuracy } \\
\hline & & & & low ctl & high ctl & low ctl & high ctl & Bias & Slope & $r$ \\
\hline$A S A$ & 0 & 1 & 1- 250 (0.999) & $\begin{array}{c}3.26 \% \\
(19)\end{array}$ & $\begin{array}{c}4.41 \% \\
(203)\end{array}$ & $\begin{array}{l}8.06 \% \\
(20)\end{array}$ & $\begin{array}{l}5.89 \% \\
(208)\end{array}$ & $-3.80 \%$ & 0.950 & 0.991 \\
\hline Alloisoleucine & 0 & 1 & 1- 250 (0.999) & $\begin{array}{c}1.04 \% \\
(11)\end{array}$ & $\begin{array}{c}1.01 \% \\
(101)\end{array}$ & $\begin{array}{c}3.83 \% \\
(11)\end{array}$ & $\begin{array}{c}3.89 \% \\
(98)\end{array}$ & $-10.05 \%$ & 0.927 & 0.998 \\
\hline
\end{tabular}

* The average analyte concentration $(\mu \mathrm{M})$ is listed in parenthesis 


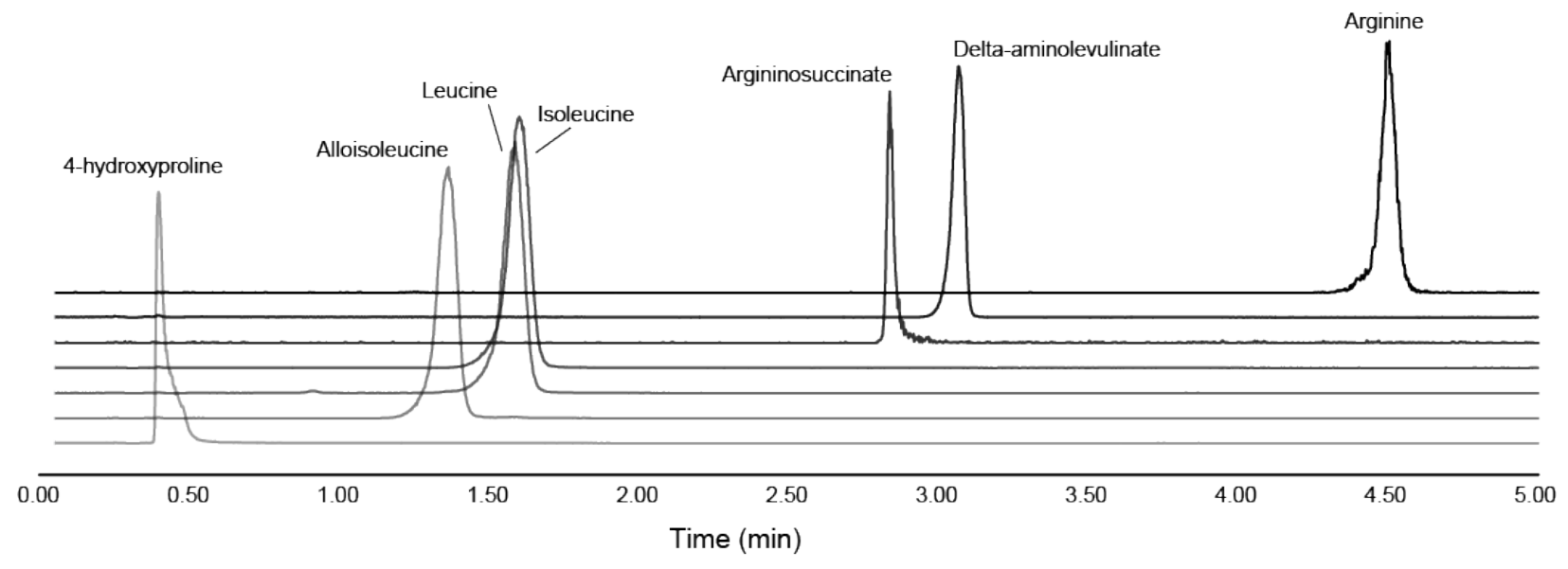

Fig. 1. Total ion chromatograms are shown for pure $75 \mu \mathrm{M}$ amino acid solutions subjected to our sample preparation and LC-MS/MS analysis protocol. 
A

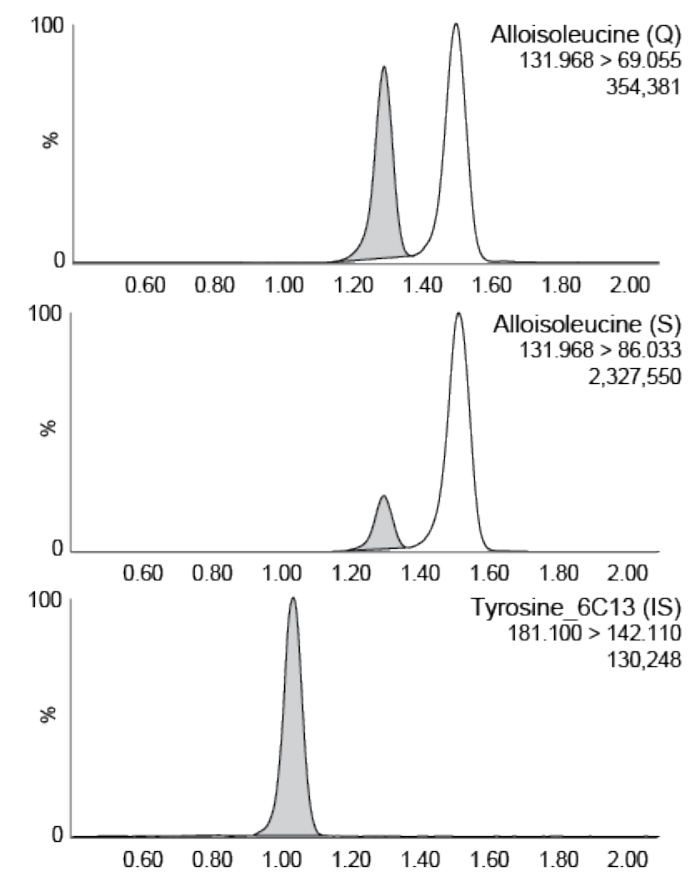

B

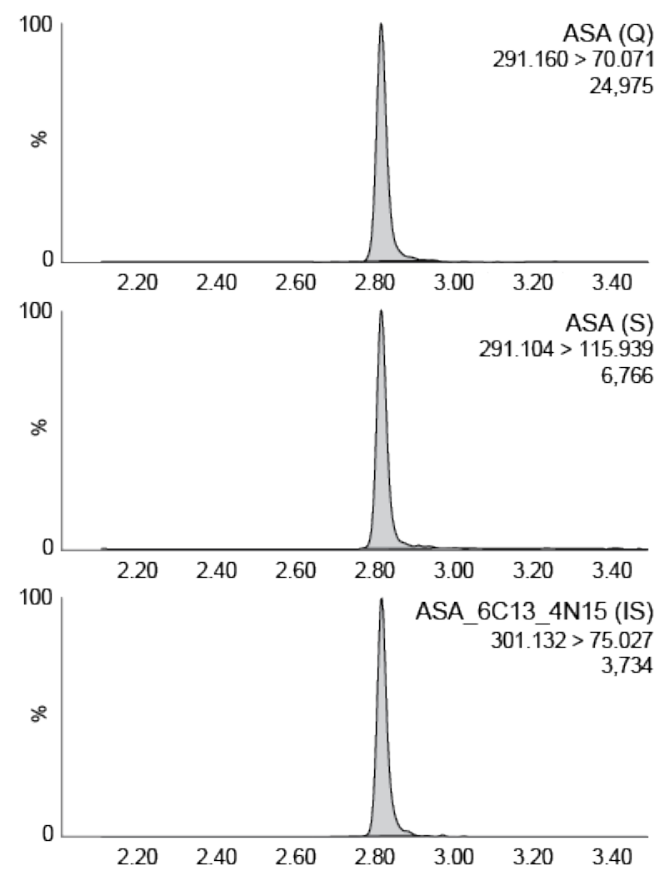

Fig. 2. Representative positive results from plasma analyses. (A) Plasma alloisoleucine concentration of $130 \mu \mathrm{M}$ in a patient with MSUD. (B) Plasma ASA concentration of $55 \mu \mathrm{M}$ in a patient with ASL deficiency. Integrated areas are shown in grey. Transitions and integrated peak areas are listed. 
A
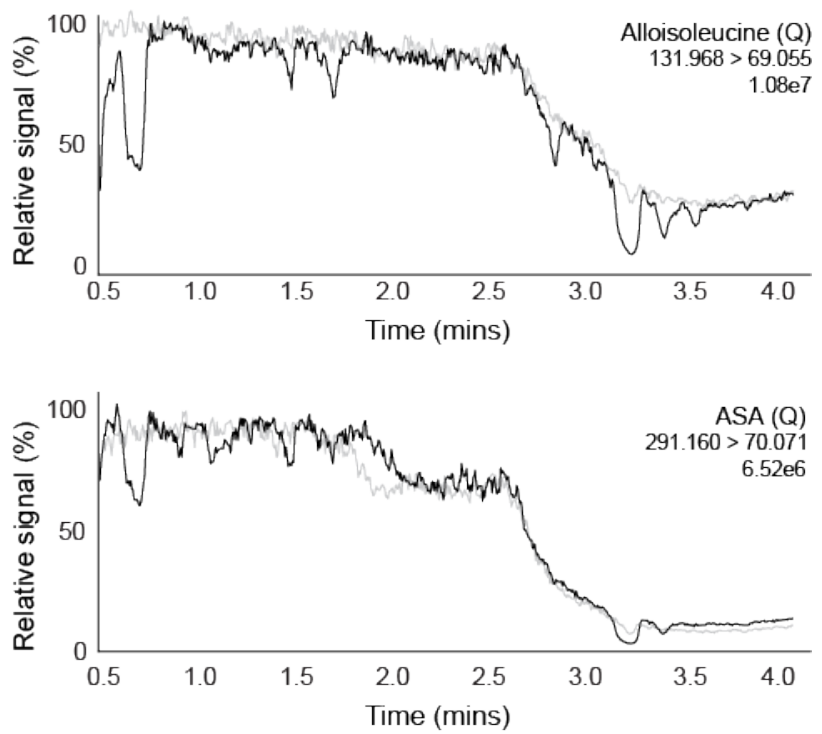

C

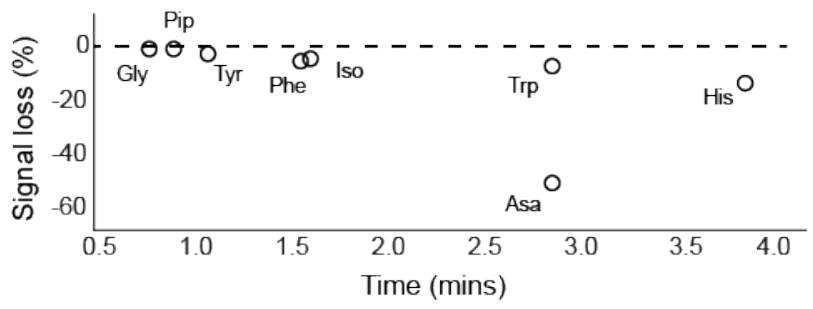

Fig. 3. Representative suppression profiles for (A) alloisoleucine and (B) ASA are shown for post column infusion studies of plasma (black line) compared to blank specimens containing only solvent A (grey line). (C) The combined effects of suppression and analyte loss were studied by monitoring spiked amino acid isotope signals in plasma vs blank specimens. Plotted values indicate the percent signal loss caused by matrix effects (calculated as $100 \times$ [(signal in plasma/ signal in blank)-1]). The dotted line indicates no difference in signal between plasma and blank. 

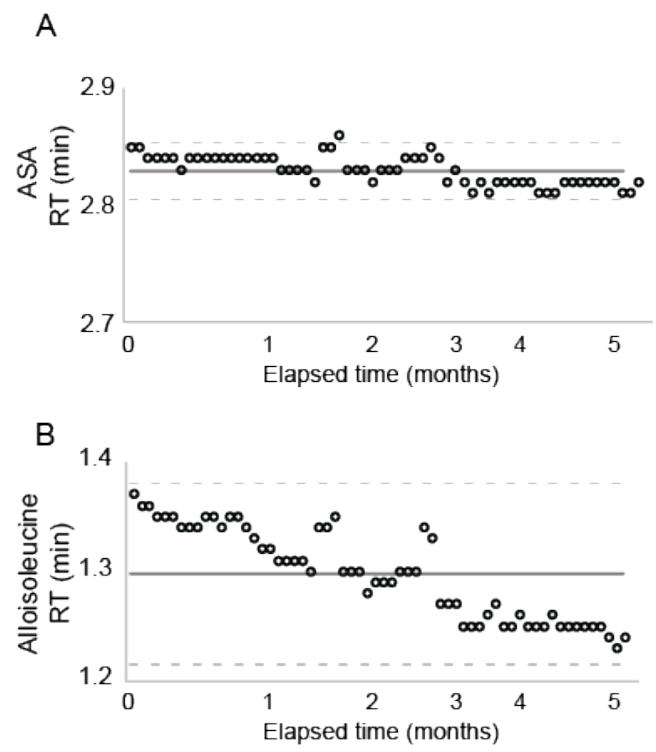

Fig. 4. Retention time Levey-Jennings plots of plasma controls are shown for (A) ASA and (B) Alloisoleucine over a period of $\sim 5$ months. Dashed lines indicate 2 standard deviations from the mean (solid line). 
A

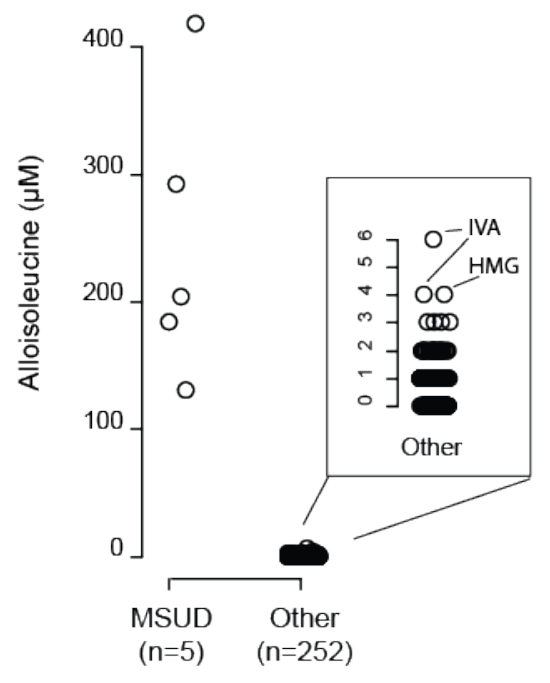

B

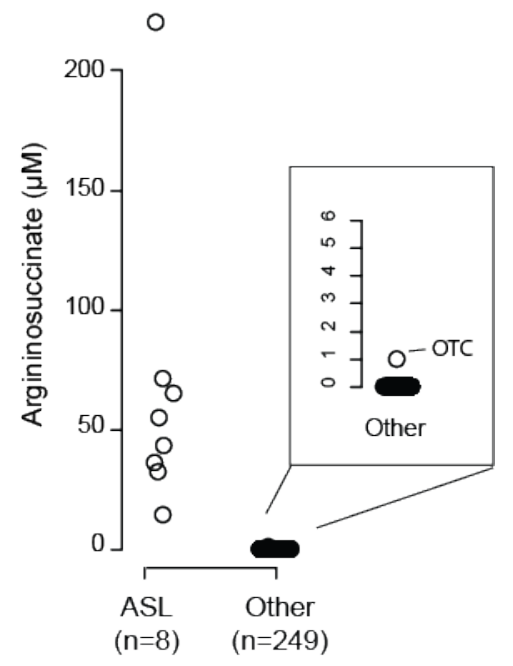

Fig. 5. Plasma (A) alloisoleucine and (B) ASA values are shown for the first 257 clinical analyses. "Other" indicates patients with no diagnosis or a diagnosis other than that being compared. Inset plots show a zoomed in view of the other subgroup. $\quad M S U D=$ maple syrup urine disease, $A S L=$ argininosuccinic lyase deficiency, IVA = isovaleric acidemia, HMG = HMG CoA lyase deficiency, OTC = ornithine transcarbamylase deficiency 


\section{References}

1. S. Moore, D.H. Spackman, and W.H. Stein, Automatic recording apparatus for use in the chromatography of amino acids, Fed Proc. 17 (1958) 1107-15.

2. D. Oglesbee, T.M. Cowan, M. Pasquali, T.C. Wood, K.E. Weck, T. Long, and G.E. Palomaki, CAP/ACMG proficiency testing for biochemical genetics laboratories: a summary of performance, Genet Med. 20 (2018) 83-90.

3. R. Filee, R. Schoos, and F. Boemer, Evaluation of physiological amino acids profiling by tandem mass spectrometry, JIMD Rep. 13 (2014) 119-28.

4. P.K. Held, L. White, and M. Pasquali, Quantitative urine amino acid analysis using liquid chromatography tandem mass spectrometry and aTRAQ (R) reagents, J Chromatogr B. 879 (2011) 2695-2703.

5. A. Le, A. Ng, T. Kwan, K. Cusmano-Ozog, and T.M. Cowan, A rapid, sensitive method for quantitative analysis of underivatized amino acids by liquid chromatography-tandem mass spectrometry (LC-MS/MS), J Chromatogr B Analyt Technol Biomed Life Sci. 944 (2014) 166-74.

6. W.A. Waterval, J.L. Scheijen, M.M. Ortmans-Ploemen, C.D. Habets-van der Poel, and J. Bierau, Quantitative UPLC-MS/MS analysis of underivatised amino acids in body fluids is a reliable tool for the diagnosis and follow-up of patients with inborn errors of metabolism, Clin Chim Acta. 407 (2009) 36-42.

7. H.C.M.T. Prinsen, B.G.M. Schiebergen-Bronkhorst, M.W. Roeleveld, J.J.M. Jans, M.G.M. de Sainvan der Velden, G. Visser, P.M. van Hasselt, and N.M. Verhoeven-Duif, Rapid quantification of underivatized amino acids in plasma by hydrophilic interaction liquid chromatography (HILIC) coupled with tandem mass-spectrometry, J Inherit Metab Dis. 39 (2016) 651-660.

8. I. De Biase, A. Liu, T. Yuzyuk, N. Longo, and M. Pasquali, Quantitative amino acid analysis by liquid chromatography-tandem mass spectrometry: implications for the diagnosis of argininosuccinic aciduria, Clin Chim Acta. 442 (2015) 73-4.

9. S.B. Narayan, G. Ditewig-Meyers, K.S. Graham, R. Scott, and M.J. Bennett, Measurement of plasma amino acids by Ultraperformance(R) Liquid Chromatography, Clin Chem Lab Med. 49 (2011) 1177-85.

10. A.P. Vilches, S.H. Norstrom, and D. Bylund, Direct analysis of free amino acids by mixed-mode chromatography with tandem mass spectrometry, J Sep Sci. 40 (2017) 1482-1492.

11. M.S. Choi, S.U. Rehman, I.S. Kim, H.J. Park, M.Y. Song, and H.H. Yoo, Development of a mixedmode chromatography with tandem mass spectrometry method for the quantitative analysis of 23 underivatized amino acids in human serum, J Pharm Biomed Anal. 145 (2017) 52-58.

12. R.G. Westall, Argininosuccinic aciduria: identification and reactions of the abnormal metabolite in a newly described form of mental disease, with some preliminary metabolic studies, Biochem J. 77 (1960) 135-44.

13. D. Rabier, P. Parvy, J. Bardet, J.M. Saudubray, and P. Kamoun, Alloisoleucine in isovaleric acidaemia, J Inherit Metab Dis. 15 (1992) 154-5.

14. S.C. Nagamani, et al., A randomized controlled trial to evaluate the effects of high-dose versus low-dose of arginine therapy on hepatic function tests in argininosuccinic aciduria, Mol Genet Metab. 107 (2012) 315-21. 